\title{
El lugar de la inteligencia empresarial en el entorno conceptual de la gestión del conocimiento. Evolución en Cuba
}

\author{
Por Eduardo Orozco Silva
}

Resumen: Se ofrece un análisis de la relación entre inteligencia empresarial y gestión del conocimiento y del vínculo de ambas con la gestión de información. Se presenta el entorno conceptual de la inteligencia empresarial y su relación con la prospectiva tecnológica. Se realiza una caracterización comparada de inteligencia empresarial y de gestión del conocimiento. Se informa brevemente sobre las actividades de inteligencia empresarial en Cuba.

Palabras clave: Inteligencia empresarial, Gestión del conocimiento, Gestión de información, Prospectiva tecnológica, Cuba.

Title: The position of business intelligence within the conceptual framework of knowledge management and its evolution in Cuba

Abstract: The relationship between business intelligence and knowledge management is analyzed, as well as their links to information management. Also explored are their relationships with technology foresighting, together with the compared characteristics of both business intelligence and knowledge management. Included in the article is a brief report on business intelligence activities in Cuba.

Keywords: Business intelligence, Knowledge management, Information management, Technology foresight, Cuba.

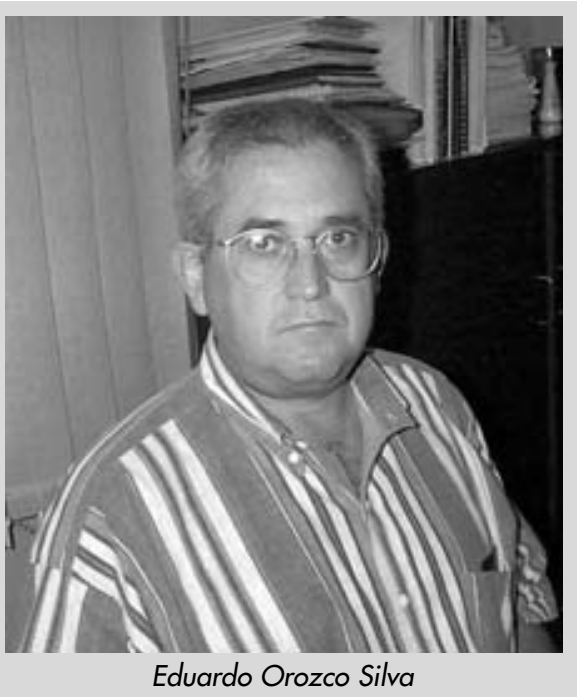

Orozco Silva, Eduardo. "El lugar de la inteligencia empresarial en el entorno conceptual de la gestión del conocimiento. Evolución en Cuba". En: El profesional de la información, 2001, julio-agosto, v. 10, n. 7-8, pp. 14-22.

\section{Introducción}

Las tecnologías de la información están facilitando herramientas cada vez más exitosas y difundidas para su empleo en la gestión de información, la inteligencia empresarial y la gestión del conocimiento. Estas aplicaciones están acercando la inteligencia empresarial a la gestión del conocimiento más de lo que se podría lograr sólo a partir de la cercanía conceptual existente y, en cierto modo, están propiciando una convergencia entre ambas. Sin embargo, a la vez se está dando una confusión creciente entre cada uno de estos conceptos, sus vínculos operacionales y la relación de ambos con la gestión de información. Aquí entenderemos ésta como el conjunto de medidas coordinadas para la selección, adquisición, almacenamiento y distribución eficientes y eficaces de los recursos de información necesarios para el trabajo de la organización. Sobre este tema hay excelentes referencias, pero se recomienda revisar a Orna (1999), que lleva a cabo su análisis incluyendo la óptica de la gestión del conocimiento.

La unión entre gestión del conocimiento y gestión de información ha sido tratada por Skyrme (1997), Ranguelov y otros autores, de modo que es innecesario hacerlo aquí. La relación entre inteligencia empresarial y gestión de la información es mucho más evidente y también ha sido tratada antes (Orozco, 2000, enero). Sin embargo, el vínculo entre inteligencia em- presarial y gestión del conocimiento no ha sido tan clarificado y por ello vale la pena dedicarle cierta atención. El objetivo del presente artículo es aportar una mínima contribución para ayudar a allanar el camino hacia la comprensión de ese nexo y, por tanto, de las formas de aplicación de la inteligencia empresarial y la gestión del conocimiento, así como dar a conocer sucintamente qué se hace en Cuba al respecto.

\section{«Gracias a la amplitud de recur- sos informáticos se está gene- rando una tendencia a asociar la inteligencia empresarial úni- camente con herramientas co- mo procesadores analíticos en línea, almacenes de datos y pro- gramas para minería de datos»}

Una revisión superficial al www arroja decenas de sitios, principalmente de carácter comercial, donde la inteligencia empresarial y la gestión del conocimiento aparecen en la primera página como estrechamente unidas por un lazo nunca identificado al adentrarse en el sitio en cuestión. Lo mismo ocurre al revisar una base de datos de publicaciones especializadas en informática: un amplio número de artículos relacionan ambos conceptos, pero sólo desde el punto de vista de 
aplicaciones informáticas, sin precisión de dónde termina uno y dónde comienza el otro. Una revisión elemental en cualquier base de datos en busca de software para inteligencia empresarial arroja cientos de ofertas (Orozco, 1999) y de artículos sobre el tema desde el punto de vista de esta disciplina. De modo similar ocurre con la gestión del conocimiento (se recomienda explorar la base de datos Computer Select, de Gale Group). En resumen, se ha escrito mucho sobre inteligencia competitiva, empresarial y organizacional. También se han publicado numerosos trabajos sobre gestión del conocimiento, pero no tanto sobre las relaciones entre estas materias.

\section{La inteligencia empresarial}

No hay una definición única para este concepto. Para el autor, es una herramienta gerencial cuya función es facilitar a las administraciones el cumplimiento de los objetivos y la misión de sus organizaciones mediante el análisis de la información relativa a su negocio y su entorno, obtenida de modo ético. Las empresas pueden implantar su propio sistema de inteligencia empresarial a partir de métodos y software propios o adquiridos de entre la amplia oferta del mercado actual. Gracias a la amplitud de recursos informáticos se está generando una tendencia a asociar la inteligencia empresarial únicamente con herramientas como procesadores analíticos en línea, almacenes de datos y programas para minería de datos, instalados costosamente en la propia organización. Sin embargo, otra opción es acudir al servicio ocasional de consultorías especializadas, que también abundan en el mercado y ayudan a las empresas a manejar la inteligencia empresarial, pero desde afuera.

En el presente trabajo se hará referencia a la inteligencia empresarial, entendida como la capacidad de reunir, analizar y diseminar datos permitiendo obtener, de manera sistemática y organizada, información relevante sobre el ambiente externo y las condiciones internas de la empresa para la toma de decisiones y la orientación estratégica; esta capacidad de las organizaciones es a la vez una función de las mismas (Orozco, 1996). Cuando el autor menciona la situación externa $\mathrm{y}$ las cuestiones internas hace referencia a todos los aspectos implicados en el desempeño de la organización: sociales, económicos, políticos y tecnológicos, tanto por separado como en su interrelación.

Al mencionar la inteligencia empresarial, se entiende como equivalente en la práctica, ya que no sinónimo exacto, de la inteligencia económica (Clerc, 1997) y de la competitiva dentro de la bibliografía anglosajona, con las correspondientes diferencias de alcance. Se percibe aquí la inteligencia económica como lo hace Clerc: "[...] la combinación de todas las medi- das coordinadas de colección, procesamiento, distribución y protección de información valiosa para los actores económicos y que se puede obtener por medios legales. Su objetivo es ofrecer a los decisores en las empresas o en el gobierno el conocimiento necesario para comprender el ambiente y ajustar las estrategias individuales o colectivas de acuerdo con el conocimiento obtenido".

«Para proyectar la política de
ciencia y tecnología en un país
se utilizan generalmente las
mismas aplicaciones que para
el impulso estratégico de una
empresa. Sin embargo, los es-
pecialistas de cada sector tra-
bajan en mundos científicos di-
ferentes. La gestión del conoci-
miento está facilitando una co-
rrelación de métodos y concep-
ciones porque se basa en lo ver-
daderamente común: el conoci-
miento»

La expresión inteligencia económica se presenta generalmente como equivalente a la competitiva, empresarial, corporativa, estratégica, etc. Ya se ha abordado el hecho de que no son sinónimos exactos (Orozco, 1999). Sin embargo, a efectos del presente trabajo es conveniente tratar otra confusión: entre inteligencia empresarial y estratégica. Por supuesto, en el ámbito de la empresa puede ser legítimo hablar de inteligencia estratégica si las aplicaciones de inteligencia empresarial se realizan sólo o principalmente para la proyección estratégica de la organización. Pero existe otro ámbito en el cual se habla de inteligencia estratégica con un sentido parcialmente diferente: el contexto de una sociedad determinada e incluso el de la sociedad en su conjunto. Es en la bibliografía sobre política científica e innovación donde se utiliza el concepto de

\section{ENTORNO CONCEPTUAL DE LA INTELIGENCIA} EMPRESARIAL

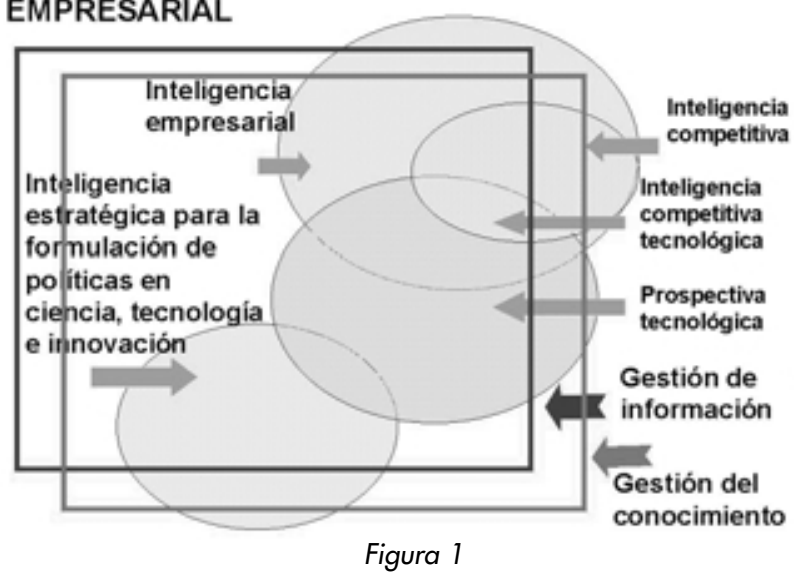


inteligencia estratégica en el sentido de " [...] tratar de analizar el comportamiento pasado, pasar revista a las opciones tecnológicas para el futuro y evaluar las consecuencias de las distintas opciones" (Kuhlmann, 2000). Este enfoque conlleva a la ejecución de la prospectiva tecnológica, que Kuhlmann entiende como "pasar revista a las opciones tecnológicas para el futuro". También se dice que el resultado principal de la prospectiva tecnológica es el "nuevo conocimiento público" relativo a los futuros posibles de las sociedades nacionales como consecuencia de los cambios tecnológicos (Technology foresight initiative, 2001).

Otras definiciones que aportan claridad a la concepción de la prospectiva tecnológica, como las de Martin (1995) e Hidalgo (1998), igualmente conllevan un proceso de estudio de información tecnológica, social, económica y de otros ámbitos para favorecer el futuro a partir de decisiones presentes. Las herramientas de análisis pueden ser tanto cualitativas como cuantitativas y, de hecho, los exámenes más completos para la prospectiva tecnológica requieren de ambas.

Es precisamente en las aplicaciones donde aparecen las similitudes y hasta los solapamientos en las técnicas de inteligencia estratégica, prospectiva tecnológica e inteligencia empresarial, con la inclusión de la inteligencia competitiva y de la inteligencia competitiva tecnológica. Sobre este último concepto se recomienda leer a Rodríguez Salvador y Escorsa Castells (1998).

\section{«En general, la inteligencia em- presarial se ejerce por grandes instituciones mediante servi- cios internos diseñados expre- samente»}

En general, la inteligencia empresarial se ejerce por grandes instituciones mediante servicios internos diseñados expresamente. No obstante, tanto grandes como medianas y pequeñas empresas acuden eventualmente a las consultorías especializadas para recibir este auxilio. Los resultados de los servicios de inteligencia empresarial, es decir, los productos de inteligencia, son generalmente del siguiente tipo:

-Perfiles estratégicos: de sector, de compañía, de producto, de personalidad.

—Estudios de mercado.

—Análisis de la competencia.

-Planes estratégicos para la introducción de la compañía o producto/servicio en un país o área.

-Evaluación de negociaciones, fusiones, adquisiciones, etc.
—Evaluación del desempeño o funcionamiento.

-Identificación de potencial oculto en competidores.

-Apoyo para planeación estratégica.

- Ayuda para estudios de benchmarking y otros.

Las técnicas para llegar a este tipo de productos han evolucionado, desde los estudios retrospectivos realizados sobre las colecciones de datos hasta la entrega de análisis prospectivos basados en minería de datos, pasando por el procesamiento analítico en línea y los almacenes de datos. En realidad, ninguna de estas técnicas de tratamiento ha desplazado a las otras, sino que se complementan según las circunstancias (v. nota).

\section{Nota}

Para un enfoque rápido y abarcador del tema, se recomienda revisar: FID review, 1999, v. 1, n. 4-5, revista publicada por la Federación Internacional de Información y Documentación. Este número fue completamente dedicado a inteligencia empresarial y se revisan técnicas de análisis en artículos que van desde el estudio de bases de datos de tesis de grado hasta el análisis de información para la prevención de riesgos en crisis económicas, incluyendo las fuentes para información sobre compañías, el papel de internet y la evolución de las técnicas de análisis de información en general.

La globalización, el desarrollo de las tecnologías de la información y la necesidad de compartir más las políticas en ciencia e innovación, han provocado una cierta convergencia de métodos y herramientas. Cuando se utilizan indicadores de ciencia y tecnología en un país para proyecciones futuras o para la corrección del rumbo en la política correspondiente, generalmente se están utilizando las mismas aplicaciones que para el impulso estratégico de una empresa. Sin embargo, los especialistas de cada sector divulgan sus experiencias en publicaciones distintas, participan en eventos científicos diferentes y se ha creado en cada caso una terminología particular. La gestión del conocimiento está facilitando una correlación de métodos y concepciones porque se basa en lo verdaderamente común entre todo lo mencionado: el conocimiento.

\section{Concepción de la gestión del conocimiento}

Ha dejado de ser una moda para convertirse en una técnica efectiva de gestión que reconoce y utiliza el valor más importante de las organizaciones: el recurso humano y el conocimiento que las personas poseen y aportan a la empresa. 


\begin{tabular}{|c|c|}
\hline Inteligencia empresarial & Gestión del con \\
\hline $\begin{array}{l}\text { Es una herramienta gerencial que mejora el } \\
\text { desempeño de modo puntual. }\end{array}$ & $\begin{array}{l}\text { Es un método de gestión que mejora el } \\
\text { comportamiento organizacional en general. }\end{array}$ \\
\hline Componente principal: información. & $\begin{array}{l}\text { Componentes } \\
\text { los recursos } \\
\text { empresarial. }\end{array}$ \\
\hline $\begin{array}{l}\text { Elemento crítico: las fuentes de } \\
\text { información y las técnicas de análisis. }\end{array}$ & $\begin{array}{l}\text { Elemento crítico: las personas como fuente } \\
\text { de información y su disposición a } \\
\text { compartir. }\end{array}$ \\
\hline $\begin{array}{l}\text { Su implantación y uso requiere de la } \\
\text { gestión de la información en la } \\
\text { organización, del empleo de las tecnologías } \\
\text { de información y de personal entrenado } \\
\text { para tareas específicas si se instaura un } \\
\text { sistema interno. }\end{array}$ & $\begin{array}{l}\text { Su implantación y uso requiere de la } \\
\text { gestión de la información en la } \\
\text { organización, de la utilización de las } \\
\text { tecnologías de información y del mejor } \\
\text { manejo de los recursos humanos en } \\
\text { general. }\end{array}$ \\
\hline \multicolumn{2}{|c|}{$\begin{array}{l}\text { No se pueden implantar aisladamente. El programa de introducción no puede ser ajeno a } \\
\text { las técnicas mencionadas arriba sino que, de hecho, debe integrarlas y en particular ese } \\
\text { programa va estrechamente unido a la gestión de la información. }\end{array}$} \\
\hline $\begin{array}{l}\text { Los servicios de inteligencia empresarial } \\
\text { pueden ser subcontratados, en dependencia } \\
\text { de los objetivos, recursos y tiempo en que } \\
\text { se necesita la respuesta. }\end{array}$ & $\begin{array}{l}\text { La gestión del conocimiento es inherente a } \\
\text { la organización. Se puede subcontratar la } \\
\text { metodologia de implantación, pero su } \\
\text { ejercicio, con conocimiento propio, ajeno o } \\
\text { con ambos, se hace dentro de la } \\
\text { organización. }\end{array}$ \\
\hline \multicolumn{2}{|c|}{$\begin{array}{l}\text { Requieren del uso de las tecnologías de información para la realización del proceso, pero } \\
\text { ellas no son componentes primarias, por encima de otros componentes. }\end{array}$} \\
\hline $\begin{array}{l}\text { Las tecnologías de información se usan } \\
\text { principalmente para la compilación y } \\
\text { análisis de la información. }\end{array}$ & $\begin{array}{l}\text { El papel de las tecnologías de información } \\
\text { se manifiesta principalmente en las } \\
\text { facilidades para compartirla. }\end{array}$ \\
\hline $\begin{array}{l}\text { La acción del sistema, se basa } \\
\text { principalmente en información cuantitativa } \\
\text { y se puede incluir el uso de información } \\
\text { cualitativa, en la forma de análisis paralelos } \\
\text { que converjan al final del proceso. }\end{array}$ & $\begin{array}{l}\text { La acción del sistema se basa } \\
\text { principalmente en información cualitativa y } \\
\text { en general se incluye el uso de información } \\
\text { cuantitativa, en dependencia de la } \\
\text { naturaleza de los procesos de la } \\
\text { organización en cuestión. }\end{array}$ \\
\hline $\begin{array}{l}\text { El resultado se usa generalmente para } \\
\text { acciones externas a la empresa. }\end{array}$ & $\begin{array}{l}\text { para acciones internas o } \\
\text { ssa, en dependencia de la } \\
\text { ón de la gestión del }\end{array}$ \\
\hline $\begin{array}{l}\text { Resultados de uso inmediato y de carácter } \\
\text { perecedero: en la práctica, lo más } \\
\text { importante es captar información para } \\
\text { convertirla en conocimiento y aplicarlo a } \\
\text { situaciones específicas. }\end{array}$ & $\begin{array}{l}\text { Resultados de uso prolongado y de carácter } \\
\text { imperecedero: en la práctica, lo más } \\
\text { importante es el flujo de conocimiento. }\end{array}$ \\
\hline $\begin{array}{l}\text { Apariencia innoble. Aunque basada en } \\
\text { métodos éticos para obtener información, } \\
\text { se ha confundido con el espionaje } \\
\text { industrial. Papel importante tanto de las } \\
\text { fuentes externas como de las internas. }\end{array}$ & $\begin{array}{l}\text { Apariencia noble. Se asume a priori como } \\
\text { la utilización de los valores propios de la } \\
\text { organización. Papel fundamental de las } \\
\text { fuentes internas, que pueden también ser } \\
\text { externas. }\end{array}$ \\
\hline
\end{tabular}


Posiblemente uno de los puntos principales de la gestión del conocimiento es su completa coherencia con cualesquiera otras técnicas, como la gestión de calidad, la reingeniería, el benchmarking y otras que, por otra parte, se basan también en el conocimiento.

Debido a su propia naturaleza, el conocimiento no se administra externamente. Sólo es posible controlar el proceso de su creación y utilización en entornos sociales, para fines de la organización. Por ello es legítimo pensar que realmente se administra la información disponible en las empresas, aunque de modo más abarcador y profundo. Sin embargo esto está en discusión entre los teóricos.

Unos dicen que la gestión del conocimiento es un nuevo enfoque de la gestión de la información; otros que es su evolución natural. Existen autores que no mencionan esa última y proponen la gestión del conocimiento como algo sustancialmente nuevo; también hay quienes la ubican dentro del campo de las tecnologías de la información. Pero es innegable que siempre hubo "gestión del conocimiento", porque constantemente las personas o las organizaciones trataron de aprender más, buscaron la forma de dejar trazas de su conocimiento (desde las pinturas en la caverna) y en la medida de su conveniencia y/o posibilidades lo compartieron.

Actualmente las tecnologías de la información permiten hacer todo lo anterior de manera sumamente eficiente, eficaz y exhaustiva. Por otra parte, los métodos de gestión empresarial cambian y justamente hoy se habla de que los enfoques de gestión, en función de elementos externos como clientes, proveedores, competidores, etc., son menos esenciales puesto que son cada vez más cambiantes. Entonces, la función más importante es la capacidad de la empresa de adaptarse a tales desarrollos. Eso se logra solamente con la organización interna adecuada, el manejo de la información, las tecnologías para su gestión y el personal preparado para el cambio. Estos elementos pasaron al liderazgo de la gestión empresarial en una combinación perfecta llamada "gestión del conocimiento". En cuanto a ésta, muchas de las definiciones caen en terreno resbaladizo y las similitudes entre ellas a veces son aparentes. De hecho lo que ocurre es que algunas son parciales, dando a la gestión del conocimiento en su conjunto el enfoque de una de sus tantas formas de expresión. Una simple ojeada a la bibliografía aporta un grupo tan amplio como diverso de descripciones. Sin embargo, algunas son suficientemente consistentes y coherentes entre sí como para basarse en ellas. A saber:

- "La gestión del conocimiento es un nuevo método de gestión organizacional que se asocia a los que se han ido desarrollando en las últimas décadas: polí- tica de innovación permanente, marketing estratégico, política de recursos humanos, dirección por objetivos, calidad total y reingeniería de procesos. La gestión del conocimiento podría resumirse en: información + gestión de recursos humanos. Se trata de desarrollar un conjunto de actuaciones y procedimientos que aporten valor añadido a las actividades de la organización y generalicen las mejores prácticas en cada uno de los procesos de su actividad" (Rodríguez Rovira, 1999).

- “[...] El concepto de gestión de conocimiento, que estaría constituido por todas aquellas actividades y procesos que permiten generar, buscar, difundir, compartir, utilizar y mantener el conocimiento de una organización con el fin de incrementar su capital intelectual y aumentar su valoración dentro de su entorno de mercado" (Lozano y otros, 2000).

- "La creación de conocimiento organizacional debe entenderse como la capacidad orgánica para generar nuevos conocimientos, difundirlos entre los miembros de una organización y materializarlos en productos, servicios y sistemas. Es la clave del proceso a través del cual las firmas innovan" (Nonaka, I.; Tackeuchi, H., 1995).

\section{«Los métodos de gestión em- presarial cambian y hoy se ha- bla de que los enfoques de ges- tión en función de elementos externos son menos esenciales puesto que son cada vez más cambiantes»}

En conjunto aportan algunas de las características más importantes de la gestión del conocimiento y nos relevan de la necesidad de una nueva definición, más bien por la comprensión de algunas de sus funciones: la gestión del conocimiento identifica y explota el creado en la organización y el adquirido en la práctica de la misma (tanto de fuentes internas como externas), generaliza las mejores prácticas, propicia el incremento del capital intelectual de la empresa y su valor de mercado, a la vez que facilita la generación de nuevos conocimientos y su materialización en productos y servicios.

¿Cómo se logra esto mediante la gestión del conocimiento? En dependencia de la misión, objetivos, estructura, valores de cada organización y sus productos y/o servicios, puede realizarse de diferentes maneras. Es decir, puede tener diferentes formas de expresión práctica y ninguna es excluyente, por lo que es posible la coexistencia de varias de ellas. La bibliografía las presenta desde diversos puntos de vista, pero varias 
preguntas que los directivos se hacen pueden ser respondidas por la gestión del conocimiento:

1. ¿Cómo aprovechar el conocimiento estructurado de la entidad? Mediante la organización y reutilización de la información generada en la propia institución: informes internos, patentes, artículos, presentaciones en congresos, en definitiva: el "flujo ascendente" de la empresa. Tan importante es conservar estos documentos y hacerlos accesibles en el futuro, como disponer de un cuerpo normativo que garantice estabilidad en los métodos para su producción y hacer recuperable no sólo el documento, sino también el camino hacia cada documento final de una investigación, del proceso de creación, etc.

2. ¿Dónde están los que más saben sobre temas críticos? Muchas veces fuera de la organización. Entonces habrá que identificarlos, facilitar el acceso a ellos invitándolos a reuniones, seminarios, organizar redes de esos expertos. Es muy fácil su formalización mediante bases de datos. Generalmente la fuente de información para ello son los mismos empleados; de lo contrario otros expertos, las bases de datos, la prensa y otras fuentes comunes.

3. ¿Quiénes saben realizar ciertas tareas? Para llegar a un producto o servicio determinado se necesitan, entre otros, recursos humanos. Éstos principalmente aportarán su conocimiento al respecto en las diversas fases de producción: investigación de mercado o científica, elementos tecnológicos, cuestiones regulatorias y capacidad organizativa. De este modo es necesario realizar el llamado "mapeo" o inventario del conocimiento existente en la organización para llegar a una meta. Cuando no se tiene en la institución se busca fuera de ella.

4. ¿Quiénes son los líderes de conocimiento? Su identificación es de gran importancia para tareas prácticas, tanto para las de proyección a mediano y largo plazo como para apoyar su gestión mediante la identificación de expertos y de otras fuentes de información.

5. ¿Se puede aprender de la práctica sólo con la práctica? El aprendizaje por la práctica es útil y conveniente, pero es un proceso generalmente largo. Por lo tanto es necesario emplear métodos de trabajo que permitan aprovechar rápida y ampliamente las lecciones que resultan del contacto con clientes, de las dificultades o éxitos en la producción o los servicios y otros. Generalmente se obtiene la información mediante entrevistas, reuniones de trabajo, informes de progreso, etc. Es lo que se conoce como "compartir las mejores prácticas".

6. ¿Cuánto vale lo que sabemos? El aparato económico de la organización debe ser capaz de manejar e incluir en sus balances el valor del capital intelectual: copyright, información no publicada, patentes, marcas y otras formas de expresión de su valor intelectual.

7. ¿Cómo utilizar el conocimiento interno y el externo para tomar decisiones en casos concretos? Mediante la compilación, análisis y síntesis de información procedente del entorno interior o de fuera de la organización, en dependencia del problema en cuestión. Un ejemplo práctico es la implantación interna y uso de sistemas de inteligencia empresarial. Depende tanto de las bases de datos internas como otras de publicaciones, patentes, información comercial y económica, orientación política y social de los eventos nacionales e internacionales y otros muchos factores.

8. ¿Pueden los productos/servicios de nuestra organización incorporar mayor valor añadido? Un centro de información, una empresa de aviación, un laboratorio farmacéutico, etc., pueden aumentar el valor de sus productos y servicios al incorporar mayor cantidad de conocimiento a los mismos, haciéndolos mejores y eventualmente más contenedores de tecnología.

La práctica de la gestión del conocimiento logra respuestas no sólo a problemas diarios sino también a cuestiones estratégicas, pero es principalmente una herramienta de gestión que logra sacar partido al conocimiento instalado en la organización para su desempeño general. Al poseer definiciones y caracterización tanto de la inteligencia empresarial como de la gestión del conocimiento, es posible establecer una comparación que propicie la mejor comprensión de los ámbitos de aplicación y alcances de cada una como se puede ver en la tabla 1.

\section{«La gestión del conocimiento logra respuestas no sólo a pro- blemas diarios sino también a cuestiones estratégicas, pero es principalmente una herra- mienta de gestión que logra sa- car partido del conocimiento instalado en la organización»}

De la tabla resulta obvio que la inteligencia empresarial puede considerarse imbricada dentro de la gestión del conocimiento, pero conservando características propias. Lo que la organización haga por desarrollar una será útil para la evolución de la otra. Aunque ambas son beneficiosas para la práctica organizacional, los empresarios pueden ver con mayor claridad, y en un periodo de tiempo más corto, los resultados de la inteligencia empresarial. De cualquier forma sus entidades estarán en mejor posición competitiva a largo plazo si desarrollan todos los aspectos de la ges- 
tión del conocimiento, incluida la inteligencia empresarial.

Después de un enfoque comparativo como el anterior, es conveniente elaborar el entorno conceptual de la inteligencia empresarial y conocer qué lugar ocupa cada una de las herramientas mencionadas en el mismo. Una expresión gráfica se muestra en la figura 1.

Como puede apreciarse, tanto la gestión del conocimiento como de la información abarcan una buena parte de las áreas de la inteligencia estratégica, la inteligencia empresarial y la prospectiva tecnológica. Éstas, a su vez, tienen enlaces y solapamientos dados por la comunidad de herramientas. La inteligencia competitiva tecnológica es el vínculo entre la prospectiva tecnológica y la inteligencia empresarial. Todas estas aplicaciones existen sólo cuando se practica la gestión de información, mediante la cual se expresa también la del conocimiento. Es decir, ambas son entornos más amplios y abarcadores que el resto de las técnicas. A la vez, tienen un alto nivel de solapamiento, pero conservan ciertas zonas de diferenciación.

\section{Evolución en Cuba}

Las posibilidades de aplicación de la inteligencia empresarial en Cuba ya han sido analizadas (Orozco, 2000, diciembre). Sobre la base de una larga lista de antecedentes relativos al desarrollo científico-técnico del país y de los avances en las infraestructuras de servicios de información y de sus tecnologías, ha sido posible llegar a la situación actual en inteligencia empresarial. Hoy día numerosas organizaciones cubanas, tanto empresariales como de investigación y desarrollo, emplean los servicios de consultorías o mecanismos propios para beneficiarse de estas técnicas en los campos importantes de la evolución económica cubana. Por otra parte, entidades extranjeras que desean penetrar en este mercado hacen uso de la inteligencia empresarial con resultados satisfactorios. La Consultoría Biomundi, Dirección de Inteligencia Corporativa del Instituto de Información Científica y Tecnológica (Idict) brinda estos servicios desde 1992. Otras organizaciones comienzan con éxito a hacerlo, tanto en la capital como en provincias. El Idict es la organización líder en el país en servicios de información y ocupa una buena posición tanto en recursos de consultoría basados en análisis de información como en consultoría organizacional a partir de la gestión tecnológica, teniendo una extensa base de tecnología de información. Debido a su larga hoja de servicios, tiene experiencia y conocimientos que compartir en todos los aspectos de su desempeño profesional.

http://www.idict.cu
Desde 1992, mediante cursos, seminarios, eventos técnicos y servicios a las empresas, la inteligencia empresarial comenzó a tener presencia en Cuba:

- Los congresos internacionales de información, Info, que el Idict organiza desde 1988, han incluido el tema de modo permanente en forma de seminarios o con talleres especiales desde 1995.

—En 1998 se celebró el Seminario cubano-espanol de inteligencia competitiva.

-Durante Info' 99 se celebró el Coloquio cubanobrasileño de inteligencia organizacional.

-Desde 1999 es tema de una lista de discusión especializada en la que han participado no sólo profesionales de información sino también empresarios.

\section{intempres@mailer.pco.cu}

—En noviembre de 2000 se celebró el I Taller nacional de inteligencia empresarial.

-En octubre de 2001 se celebrará el II Taller de inteligencia empresarial y gestión del conocimiento en la empresa.

http://www.idict.cu/eventos2001/intempres.htm intempres2001@biomundi.inf.cu

La inteligencia empresarial ha sido asignatura de diplomados y maestrías en gestión de información, y en septiembre del presente año comenzará a impartirse la especialización en inteligencia empresarial.

\section{«En la bibliografía anglosajona la inteligencia empresarial se entiende en la práctica como equivalente de la inteligencia económica y de la competitiva (no como sinónimo exacto)»}

Con respecto a gestión del conocimiento, son varias las organizaciones que trabajan en el tema y lo promueven. Gecyt, la empresa de Gestión del Conocimiento y las Tecnologías, lo ha incluido con éxito en su encuentro nacional sobre gestión tecnológica así como en los encuentros provinciales y en particular en Ibergecyt, el Seminario iberoamericano sobre tendencias modernas en gerencia de ciencia e innovación tecnológica.

El Idict está realizando los estudios previos para desarrollar su modelo de gestión del conocimiento, tanto en la sede central como en las trece filiales provinciales.

El Plan general de desarrollo de la gestión del conocimiento en el Idict contempla los siguientes aspectos: 
-Divulgación y culturización sobre gestión del conocimiento dentro y fuera del sistema de la institución.

-Formación de personal interno y externo en gestión del conocimiento.

-Perfeccionamiento del uso de las herramientas de gestión de información para propiciar y ejecutar la gestión del conocimiento en el Idict.

-Evaluación de software para gestión de la información y del conocimiento útiles en el instituto.

- Sistema permanente de inteligencia empresarial que contribuya a la gestión del conocimiento y a las decisiones en el sistema del Idict.

\section{- Plan piloto para la introducción de la gestión del} conocimiento en el sistema del instituto.

-Establecimiento del modelo de gestión del conocimiento en el Idict.

- Instauración de servicios de gestión del conocimiento.

\section{Conclusiones}

La inteligencia empresarial y la gestión del conocimiento tienen más similitudes que diferencias. La gestión de la información es el marco práctico sobre el cual se realizan tanto la primera como la segunda. Sin información no hay inteligencia; sin administrar la información no se puede gestionar el conocimiento. La convergencia entre inteligencia empresarial y gestión del conocimiento no significa la anulación de alguna de ellas sino más bien el fortalecimiento de ambas, a partir del empleo de técnicas comunes y de la visualización en la práctica de las ventajas de una y de otra. Estas virtudes comienzan también a hacerse visibles en Cuba, donde tanto empresas como centros de investigación y desarrollo utilizan estas técnicas para su mejor desempeño.

\section{Bibliografía}

Clerc, P. "Economic intelligence". En: World information report, 19971998, Unesco, Paris, pp. 304-317.

Computer Select. Base de datos sobre tecnologías de la información. Producida por Gale Group, 362 Lakeside Drive, Foster City, CA 94404, California, EUA. Consultada en disco compacto: enero-diciembre, 2000. http://www.galegroup.com

FID-International Federation for Information and Documentation, P. O. Box 90402, 2509, LK The Hague, The Netherlands.

http://www.fid.nl

fid@fid.nl

Hidalgo Nuchera, A. "Organización y gestión de la innovación tecnológica”. En: Manual del curso organización y gestión de la innovación tecnológica. Madrid: Universidad Politécnica de Madrid, 1998.

http://www.skyrme.com/pubs/on97full.htm
Kuhlmann, S. Inteligencia distribuida: cómo combinar la evaluación política, la prospectiva y la evaluación tecnológica. Consultado el: 17-01-00. http//www.jrc.es/pages/iptsreport/vol40/spanish/RTD35406.htm

Lozano Quirce, M. M.; Sevilla Escribano, A.; Valtueña Rincón, R. "Aproximación a la gestión del conocimiento. Una visión práctica”. En: Gerencia informática de la Seguridad Social (Giss). Consultado el: 2101-01.

http://www.gestiondelconocimiento.com

Martin, B. "Foresight in science and technology". En: Technology analysis \& strategic management, 1995, v. 7, n. 2, p. 140. Citado por Kuhlman, S. "Inteligencia distribuida: cómo combinar la evaluación política, la prospectiva y la evaluación tecnológica”. Consultado el: 17-01-00.

http//www.jrc.es/pages/iptsreport/vol40/spanish/RTD35406.htm

Nonaka, I.; Tackeuchi, H. "The knowledge creating company". Oxford University Press, 1995. Citado por Lara, J. L. "Diez respuestas a las preguntas más frecuentes sobre gestión del conocimiento", Instituto Nacional de Estadística y Censos de Argentina. Consultado el: 21-01-01.

http://www.gestiondelconocimiento.com

Orna, E. Practical information policies. $2^{\underline{a}}$ ed. Hampshire: Gower, 1999, p. 375 . Isbn 0566076934.

Orozco Silva, E. Documento no publicado entregado a los participantes en el curso: "La inteligencia corporativa para la toma de decisiones en la organización moderna”. Dictado en el Centro de Información Tecnológica, San José, Costa Rica, 1996, marzo, 150 p.

Orozco Silva, E. "La inteligencia organizacional en la industria biofarmacéutica”. En: Ciência da informação, 1999, enero-abril, v. 28, n. 1, pp. 59-66.

Orozco Silva, E. "El papel de la prospectiva tecnológica en la inteligencia empresarial. Situación en Cuba". En: IV Encuentro iberoamericano de estudios prospectivos, 2000.

Orozco Silva, E. "Competitive intelligence in Cuba: myth, reality and perspective". En: Competitive intelligence review, 2000, diciembre, v. 11, n. 4 .

Ranguelov Yulianov, S. "Gestión de la información como elemento de la gestión del conocimiento". Consultado el: 21-01-01.

http://gestiondelconocimiento.com/documentos2/stanislav_ranguelov/info.htm

Rodríguez Salvador, M.; Escorsa Castells, P. "Transformación de la información a la inteligencia tecnológica en la organización empresarial: instrumento para la toma de decisiones estratégicas". En: Recitec, 1998, v. 2, n. 3, pp. 177-202. Consultado en: abril, 1999.

http://www.fundaj.gov.br

Rodríguez Rovira, J. M. "La gestión del conocimiento: una gran oportunidad". En: El profesional de la información, 1999, marzo, v. 8, n. 3, pp. 4-7.

Skyrme, D. J. "From information management to knowledge management: are you prepared?”. En: OnLine ‘97. Consultado el: 01-03-01. http://www.skyrme.com/pubs/on97full.htm

Technology foresight initiative-abstract. Technology foresight for Latin America. United Nations Industrial and Development Organization (Unido)/International Centre for Science and High Technology, Trieste, Italia. Consultado en: 02-03-01.

http://www.foresight.ics.trieste.it/Technology_Foresight/abstract.htm 